\title{
CONTROL THEORY AND FAST MARCHING TECHNIQUES FOR BRAIN CONNECTIVITY MAPPING
}

\author{
Emmanuel Prados ${ }^{1} \quad$ Christophe Lenglet ${ }^{2} \quad$ Jean-Philippe Pons ${ }^{2}$ \\ Nicolas Wotawa ${ }^{2}$ Rachid Deriche Olivier Faugeras $^{2} \quad$ Stefano Soatto $^{1}$ \\ ${ }^{1}$ UCLA Vision Lab., USA — $\quad{ }^{2}$ Odyssée Lab., INRIA, France
}

\begin{abstract}
We propose a novel, fast and robust technique for the computation of anatomical connectivity in the brain. Our approach exploits the information provided by Diffusion Tensor Magnetic Resonance Imaging (or DTI) and models the white matter by using Riemannian geometry and control theory. We show that it is possible, from a region of interest, to compute the geodesic distance to any other point and the associated optimal vector field. The latter can be used to trace shortest paths coinciding with neural fiber bundles. We also demonstrate that no explicit computation of those 3D curves is necessary to assess the degree of connectivity of the region of interest with the rest of the brain. We finally introduce a general local connectivity measure whose statistics along the optimal paths may be used to evaluate the degree of connectivity of any pair of voxels. All those quantities can be computed simultaneously in a Fast Marching framework, directly yielding the connectivity maps. Apart from being extremely fast, this method has other advantages such as the strict respect of the convoluted geometry of white matter, the fact that it is parameter-free, and its robustness to noise. We illustrate our technique by showing results on real and synthetic datasets. Our GCM (Geodesic Connectivity Mapping) algorithm is implemented in $\mathrm{C}++$ and will be soon available on the web.
\end{abstract}

\section{INTRODUCTION}

Diffusion magnetic resonance imaging [7] is a technique to characterize the anisotropic diffusion of water molecules in structured biological tissues. As of today, it is the only non-invasive method that allows to distinguish the anatomical structures within the cerebral white matter. Diffusion tensor (DT) imaging [4] models the probability density function of the three-dimensional molecular motion, at each voxel of a DT image, by a local Gaussian process whose covariance matrix is precisely given by the diffusion tensor. Among other applications, diffusion tensor imaging (DTI) is extremely use- ful to estimate the anatomical connectivity of the human brain.

Following [19], various local approaches have already been proposed to tackle this problem. They are based on line propagation techniques and rely on the fact that the eigenvector of the diffusion tensor associated to the major eigenvalue, provides a relatively accurate estimate of the fibers' orientation at each voxel. These methods may be refined to incorporate some natural constraints such as regularity or local uncertainty and avoid being stopped in regions of low anisotropy $[5,33,8,13,16]$. All these efforts aim to overcome the intrinsic ambiguity of diffusion tensor data arising from partial volume effects at locations of fiber crossings [2]. They provide relatively accurate models of the white matter macroscopic bundles.

Most recent work can be divided into approaches based on Bayesian models and geometric methods, the latter essentially based on front-propagation techniques. They are both more robust to noise and partial volume effects than previous work, and naturally yield probability/scalar measures which can be used to evaluate the degree of connectivity between voxels. In $[6,22,12]$ stochastic tractography algorithms were introduced by modeling the uncertainty of the local fiber orientation. Through uncertainty propagation, they provide a powerful means to evaluate the probability of connection between points of the white matter. However, the intrinsic drawback of these methods is their computational complexity since it is necessary to resort to Markov Chain Monte Carlo methods or, as in [12], to evaluate probability density functions at enough locations of the space of interest.

Geometric methods use either Level Set methods [20, 17, 21], Fast Marching methods [23, 32, 28] or iterative sweeping techniques [14] to evolve a front on the basis of the diffusion tensor directional information. As described in [9], it is possible to adapt the Level Set-based front propagation technique to take advantage of the information provided by high angular resolution diffusion MRI. However, this method tends to 
be somewhat inefficient since, even with a narrow-band implementation, the number of points where the evolution speed has to be evaluated greatly increases as the surface grows. We will also show that this class of methods is prone to interpolation errors at the boundary of the domain. For our brain connectivity problem, this may lead to erroneous connections in highly convoluted areas.

Our contribution is threefold: First of all, on the basis of [17], we propose to efficiently and robustly estimate the anatomical connections of the white matter as geodesics in $\mathbb{R}^{3}$ equipped with a Riemannian metric derived from the diffusion tensor. We demonstrate that it is possible to solve, quickly and simultaneously, for the geodesic distance, the optimal vector field (optimal dynamics) corresponding to the geodesics velocities and the statistics, along those curves, of a local connectivity measure. To our knowledge, the proposed GCM algorithm is faster than any other existing method. Also, contrary to other approaches, we simply solve the anisotropic eikonal equation and do not resort to any anisotropy related parameter to constrain the front propagation. The second contribution is the ability of the algorithm to work within a mask of the white matter accurately obtained by segmentation of a high-resolution anatomical MRI. As we will show, this is crucial for the application of interest since we must strictly respect the geometry of the cortical foldings or white matter / cerebrospinal fluid (CSF) interface to recover meaningful connections. To our knowledge this technical issue has never been addressed before. Finally, for a region of interest $x_{0}$ (i.e. a point of the white matter), our GCM method generates statistics of a local connectivity measure along the geodesics linking $x_{0}$ to other locations of the brain. This can be used to discriminate likely and unlikely connections.

\section{FROM GEOMETRY TO CONTROL THEORY}

In $[20,17]$, the problem is formulated in the framework of Riemannian geometry. The white matter is interpreted as a Riemannian manifold and the diffusion tensor provides the Riemannian metric, which in turn determines white matter fibers as geodesic paths. We remind the basic definition of geodesics for convenience [11].

Definition 2.1 (geodesics) Let $\left(\mathcal{M},|\cdot|_{R}\right)$ be a Riemannian manifold. Let $x, y \in \mathcal{M}$. The geodesic connecting $x$ to $y$ is the curve $\gamma_{0}$ which minimizes the arc length, i.e.

$$
\gamma_{0}=\arg \min _{\gamma \in \Gamma x, y} \int_{0}^{T_{x y}}\left|\gamma^{\prime}(t)\right|_{R} d t
$$

where $\Gamma x, y$ is the set of curves $\gamma:\left[0, T_{x y}\right] \rightarrow \mathcal{M}$ such that $\gamma(0)=x, \gamma\left(T_{x y}\right)=y$ and $\left|\gamma^{\prime}(t)\right|_{R}=1$.

In [17], the authors show that the appropriate metric to our problem is the one associated to the norm $|\cdot|_{R}$ defined by $|x|_{R}=\sqrt{x^{T} D_{x}^{-1} x}$, where $D_{x}$ is the symmetric positive definite $3 \times 3$-matrix given by the measured diffusion tensor, i.e. the data. Let us also denote with $A_{x}$ the (symmetric positive definite) square root matrix of $D_{x}$ and with $|\cdot|_{E}$ the Euclidian norm. Let us note that we have trivially $|x|_{R}=\left|A_{x}^{-1} x\right|_{E}$.

Here, rather than interpreting the problem in terms of Riemannian geometry, we adopt an optimal control point of view. The two interpretations are equivalent, but focus on different aspects of the problem. In the Riemannian setting, the emphasis is on the description of the geometry and in particular on the geodesics. In the optimal control interpretation, the emphasis is on the optimal dynamics, i.e. the intrinsic gradient of the distance function or, in other words, the vector field tangent to the geodesics. Specifically, let a domain $\Omega$ be a subset of $\mathbb{R}^{3}$ representing the white matter. We consider the set $\mathcal{A}$ (compact subset of $\mathbb{R}^{N}$ ) of admissible controls $a$ $\left(a \in \mathcal{A}\right.$ ), a target (here the point of interest $x_{0}$, origin of the distance function), a vector field $f(x, a)$ (called dynamics) that depends on the control and a cost $l(x, a), x \in \Omega$. We call control function, a function $\alpha():. \Omega \rightarrow \mathcal{A}$. Under some regularity assumptions, to each control function $\alpha$ and $x \in \Omega$, we can associate a single trajectory $\xi_{x, \alpha}(t) \in \Omega$ following the dynamics $\xi^{\prime}(t)=f(\xi(t), \alpha(\xi(t))), t>0$, imposed by the control $\alpha$, see [3]. Moreover, one can prove that there exists a control function $\alpha^{*}$ (the optimal control) such that for all $x$, the integral of the cost along the associated trajectory $\xi_{x, \alpha^{*}}$ is minimal [3]. We then denote by

$$
\xi_{x}^{*} \stackrel{\text { def }}{=} \xi_{x, \alpha^{*}}
$$

the optimal trajectory starting from $x$ and

$$
f_{x}^{*} \stackrel{\text { def }}{=} f\left(x, \alpha^{*}(x)\right)
$$

the optimal dynamics at $x$. The goal is to characterize and compute this optimal control $\alpha^{*}\left(f_{x}^{*}\right.$ being immediately deduced from $\left.\alpha^{*}\right)$.

If we let $l(x, a)=1$, then the problem consists in finding the control function $\alpha^{*}$ s.t. for all $x$ in $\Omega$ and for all $\alpha$

$$
\begin{aligned}
\int_{0}^{T_{x, x_{0}, \alpha^{*}}} l\left(\xi_{x, \alpha^{*}}\right. & \left.(t), \alpha^{*}\left(\xi_{x, \alpha^{*}}(t)\right)\right) d t \\
& \leq \int_{0}^{T_{x, x_{0}, \alpha}} l\left(\xi_{x, \alpha}(t), \alpha\left(\xi_{x, \alpha}(t)\right)\right) d t
\end{aligned}
$$

i.e.

$$
T_{x, x_{0}, \alpha^{*}} \leq T_{x, x_{0}, \alpha},
$$

where $T_{x, x_{0}, \alpha}$ is the first time for which the trajectory $\xi_{x, \alpha}$ (controlled by the dynamics $f$ ) reaches the target $x_{0}$. $T_{x, x_{0}, \alpha}=+\infty$ if the trajectory does not reache $x_{0}$. In other words (misusing the notations) $\alpha^{*}$ is

$$
\begin{aligned}
\alpha^{*} & =\arg \min _{\alpha(.)}\left\{\int_{0}^{T_{x, x_{0}, \alpha}} l(\xi(t), \alpha(\xi(t))) d t\right\} \\
& =\arg \min _{\alpha(.)}\left\{\int_{0}^{T_{x, x_{0}, \alpha}} 1 d t\right\}=\arg \min _{\alpha(.)}\left\{T_{x, x_{0}, \alpha}\right\} .
\end{aligned}
$$

If furthermore we let $\mathcal{A}$ be the set of $A_{x}^{T} b$ for $b$ in the Euclidian unit sphere $(\mathcal{A}$ coincides then with the unit Riemannian 
sphere associated to $\left.A_{x}\right)$ and $f(x, a)=a$ (i.e. the dynamics is equal to the control), then we can see that in this case, the optimal trajectories $\xi_{x}^{*}$ correspond to the geodesics considered in [17] (when $\alpha$ covers $\mathcal{A}, \xi^{\prime}(t)=\alpha(\xi(t))$ also covers $\mathcal{A}$ ).

The control interpretation has distinct advantages: All the objects of interest live in $\mathbb{R}^{3}$ (instead of a manifold), and are governed by the Euclidian metric, hence the interpretation is independent of the geometric structure. As an illustration of this benefit, in order to estimate the direction of the geodesics, Lenglet et al. [17] proposed to compute the gradient of the distance function on the manifold, which requires some care in order to take into account the geometry imposed by the metric and is a challenging task when working on an irregular domain such as the brain white matter. In the control formalism the interpretation is rather direct: the tangent of the geodesics is in fact the optimal dynamics $f_{x}^{*}$ (since the geodesic corresponds to the optimal trajectories). Also, the optimal dynamics $f_{x}^{*}$ coincides with the optimal control, which is the direct outcome of our algorithm. The control framework reveals the fact that the value function $V$ defined by the min of equation (2)

$$
\begin{aligned}
V(x)=\min _{\alpha}\left\{\int_{0}^{T_{x, x_{0}, \alpha}} l(\xi(t), \alpha(\xi(t))) d t\right\} \\
\quad=\min _{\alpha}\left\{\int_{0}^{T_{x, x_{0}, \alpha}} 1 d t\right\}=\min _{\alpha} T_{x, x_{0}, \alpha}
\end{aligned}
$$

is the viscosity solution of the partial differential equation (PDE)

$$
\sup _{a \in \mathcal{A}}\{-f(x, a) \cdot \nabla u(x)-l(x, a)\}=0,
$$

verifying $u\left(x_{0}\right)=0$ and complemented by state constraints on the boundary of the domain $\partial \Omega[3,30]$ (let us remind that the domain is here the white matter). Also, the reader can easily verify that this function $V$ coincides with the Riemannian distance to $x_{0}$ under the metric $|.|_{R}$. An explicit Hamiltonian associated to this PDE is

$$
H_{A E i k}(x, p)=\left|A_{x} p\right|_{E}-1=|p|_{R}-1 \text {. }
$$

The control framework [3] also reveals that $f\left(x, \alpha_{x}^{*}\right)=$ $-\nabla H(x, \nabla u(x))$ where $H$ is the Hamiltonian associated to the PDE (4) and $\nabla u$ is the gradient of its solution. Finally, the control formulation of the problem directly yields our numerical method, which we report in sections 4.2 and 4.3. For practical purposes, we will adopt either interpretation depending on the situation and exploit their complementary benefits.

\section{CONNECTIVITY MEASURES}

We start by pointing out that, for a fixed point $x_{0}$ and any point $x$, the geodesic $\gamma_{x}$ (associated to the metric given by the tensors) connecting $x$ to $x_{0}$ always exists. If $x$ is connected to $x_{0}$ by a white matter fiber then the associated geodesic $\gamma_{x}$ coincides with the fiber. Nevertheless, for any $x$, the associated geodesic $\gamma_{x}$ does not necessarily coincide with a fiber. Also, in order to reconstruct the white matter fibers, we then need to be able to trace the geodesics and to evaluate if a point is potentially connected to $x_{0}$.

In this section, we propose a score to measure the expectation that a given geodesic truly represents the connection of a point $x$ with $x_{0}$. By computing statistical maps of this measure for all points $x$ in the brain, we can then determine which points are likely to be connected to $x_{0}$ and then trace the fibers. In section 4 we propose an original numerical scheme based on Fast Marching methods (FMM) to efficiently compute these maps.

Let us fix a point of interest $x_{0} \in \bar{\Omega}$ and let us consider the PDE/control/Riemannian problem associated with DTI. In section 2 , we show that, $\forall x \in \Omega$, the optimal dynamics $f_{x}^{*}$ coincides with the derivatives of the geodesics $\gamma^{\prime}(t)$ at $x$ and that they are in the Riemannian unit ball $B_{R}(0,1)$ which is also the set $\left\{A_{x} q, q \in B_{E}(0,1)\right\}$.

So, for a fixed point $x$ (and a fixed tensor $D_{x}$ ), the larger the Euclidian norm of $f_{x}^{*}$, the more confident we are in the local direction of the geodesic. Following this idea, we then define a general (local) confidence measure:

$$
\mathcal{C}(x)=\sqrt{f_{x}^{* T} D_{x}^{\alpha} f_{x}^{*}}
$$

$\alpha$ being in $\mathbb{R}$. In addition to being intuitive, this measure inherits the robustness to noise of the optimal dynamics. It also exploits the full information provided by the diffusion tensor. Finally, it does not penalize any direction in case of isotropy. Let us now discuss the possible values of $\alpha$ : if $\alpha=-1$, we get $\mathcal{C}(x)=1, \forall x \in \bar{\Omega}$. This simply means that, when we use the Riemannian metric given by the inverse of the diffusion tensor, all the geodesics are equivalent. On the contrary, when $\alpha=0$, we have $\mathcal{C}(x)=\left|f_{x}^{*}\right|_{E}$ and we claim that it is a natural local measure of connectivity since this measures the speed of propagation at $x$. Finally, when $\alpha \rightarrow \infty$, this boils down to considering the alignment of the optimal dynamics with the local major eigenvector. This was used in [23] but it is highly sensitive to isotropic areas where, by definition, the major eigenvector is undefined.

From this local connectivity measure, we can define global information from its statistics (mean and standard deviation) along the optimal trajectory:

$$
\begin{gathered}
\mu(x)=\langle\mathcal{C}(x)\rangle=\frac{1}{\tau_{x}^{*}} \int_{0}^{\tau_{x}^{*}} \mathcal{C}\left(\xi_{x}^{*}(t)\right) d t, \\
\sigma(x)=\sqrt{\left\langle\mathcal{C}(x)^{2}\right\rangle-\langle\mathcal{C}(x)\rangle^{2}} .
\end{gathered}
$$

where $\tau_{x}^{*}$ is the length of the optimal trajectory $\xi_{x}^{*}$. We should point out that, since $\left|\xi_{x}^{* \prime}\right|_{R}=1$, this length (i.e. the geodesic distance between the curve endpoints $x_{0}$ and $x$ ) coincides with the arrival time $T_{x, x_{0}}$ introduced in section 2 .

A point $x$ connected to $x_{0}$ by a white matter fiber will have a large value for $\mu(x)$ and a small standard variation $\sigma(x)$. 
The choice of using the mean instead of just integrating along the trajectories allows the comparison of two points $x$ and $y$ which are located at different distance from $x_{0}$, i.e. s.t. $\tau_{x}^{*} \neq \tau_{y}^{*}$. Although the mean value of the connectivity may be sufficient to discriminate likely fibers, the variance of this quantity may also be of great help since an ideal fiber would exhibit a high coherence of $\mathcal{C}(x)$ along its trajectory.

Let us remark that to compute the optimal dynamics, we need the geodesic distance $\tau_{x}^{*}$. In practice, we just need to compute

$$
\mathcal{R}(x)=\int_{0}^{\tau_{x}^{*}} \mathcal{C}\left(\xi_{x}^{*}(t)\right) d t
$$

and

$$
\mathcal{S}(x)=\int_{0}^{\tau_{x}^{*}} \mathcal{C}\left(\xi_{x}^{*}(t)\right)^{2} d t
$$

The values of $\mu(x)$ and $\sigma(x)$ are then derived immediately.

\section{A FAST NUMERICAL ALGORITHM}

\subsection{Related Work and Contributions}

To the best of our knowledge, there is no algorithm to compute directly the geodesics or a fiber connectivity confidence map to a point $x$ from DTI data. All the methods recovering white matter fibers proceed by implementing successively the following four steps:

1. computation of the distance function to $x$,

2. extraction of the gradients of the distance function,

3. estimation of the optimal dynamics from the gradients of the distance function,

4. tracing of the geodesics from the computed directions. This last step needs in particular an interpolation of the derivatives of the geodesics.

Some slight variants are proposed in the literature (see [10, 15] and references therein).

We wish to emphasize that the explicit tracing of the geodesics is a prerequisite to all the previous methods for computing connectivity confidence measures which in fact consist in the integration of a local criterion along the entire geodesic during the geodesics tracing step. Thus, the estimation of a complete map of connectivity measures needs to explicitly trace all the geodesics starting from all the points of the map. This approach is rather computationally intensive.

The numerical method we propose here for computing the confidence measures does not need to trace any geodesic. The confidence measure map is a direct output of our algorithm. It simultaneously and consistently computes the (geodesic) distance function, the optimal dynamics and the confidence measures.

The methods of the type "Fast Marching" [32, 28, 29, 26] are "one-pass" methods allowing to solve numerically partial differential equations of the type (4). Based on a causality principle, the Fast Marching Methods (FMM) stand in contrast to iterative methods (see for example [27, 31] and more specifically [14] in our field) which iteratively update the approximations of the solution by using paths that do not depend on the data. The idea of the FMM consists in computing the solution of the PDE in proportion as a front propagates along the optimal trajectories. Our algorithm extends the classical FMM $[32,28,29,26]$ by computing and returning in addition the optimal dynamics and the connectivity confidence measures. The consistency of our results relies on the fact that for all the computations we use the same (optimal) simplex.

Let us remark that all the quantities we compute are essential: The optimal dynamics are necessary in order to trace the geodesic, which in turn is useful for the visualisation of the fibers. Even if the result of the computation of the (geodesic) distance is not required for tracing the geodesic, it is essential for obtaining the final measures (expectation and standard deviation) we use in practice to estimate the connectivity confidence. In other respects, since our method is a "one pass method" based on front propagation, we do not need to wait for the complete computation of the distance function on the whole domain to be able to exploit it for computing the connectivity measures.

In the sequel, we describe our global algorithm and then the implementation of each specific step.

\subsection{Global Algorithm}

As in the classical "Fast Marching Method" [28, 29, 26], the grid points are divided into the three classes: Accepted, Considered, Far. Below $U, f, R$ and $S$ are respectively the approximations of the (geodesic) distance function, the optimal dynamics $f_{x}^{*}, \mathcal{R}$ and $\mathcal{S}$ (defined in section 3). $x_{0}$ is the interest point. The overall algorithm is described below:

\footnotetext{
$\overline{\text { Algorithm } 1 \text { Fast Marching algorithm for the computation of }}$ $U, f, R$ and $S$

1: Start with all the grid points in Far.

2: Move $x_{0}$ and the grid points on the boundary $\partial \Omega$ to $A c$ cepted. Set $U\left(x_{0}\right)=0$ and $U(x)=+\infty$ (FLT_MAX in practice) for all $x \in \partial \Omega$.

3: Move all the grid points adjacent to the Accepted points into Considered and for such points $x$, evaluate $U(x)$ by using the update scheme (5) and modify the associated optimal dynamics to $f(x)$; see section 4.3.

4: Find the Considered point $\tilde{x}$ with the smallest value $U(x)$. Move $\tilde{x}$ from Considered to Accepted. Compute and assign $R(\tilde{x})$ and $S(\tilde{x})$, see section 4.4.

5: Move from Far into Considered, all the Far points which are adjacent to $\tilde{x}$.

6: Re-evaluate $U(x)$ and the associated dynamics $f(x)$ for all the Considered points adjacent to $\tilde{x}$, see section 4.3.

7: If the set Considered points is not empty, return to step 4.
} 


\subsection{Distance and Optimal Dynamics Computation}

Because of space limitation, here, we only shortly describe the updating step returning the approximation of the distance function and the optimal dynamics. A complete description of its implementation can be found in our technical report [25]. Let us also remind that our $\mathrm{C}++$ code is going to be freely distributed. Following [26], we use the scheme

$$
S(\rho, x, t, u)=\sup _{a \in A}\left\{-f(x, a) \cdot P_{s_{1}(x, a), . ., s_{N}(x, a)}-l(x, a)\right\}
$$

where the $i^{\text {th }}$ component of $P_{s_{1}, . ., s_{N}}$ is given by

$$
\begin{aligned}
{\left[P_{s_{1}, . ., s_{N}}\right]_{i} } & =\frac{t-u\left(x+s_{i} h_{i} e_{i}\right)}{-s_{i} h_{i}}, \\
s_{i}(x, a) & =\operatorname{sign}\left(f_{i}(x, a)\right),
\end{aligned}
$$

$h_{i}$ denotes the grid size in the $i^{\text {th }}$ direction and $\left\{e_{i}\right\}$ is the canonical basis of $\mathbb{R}^{N}$. In our case, $N=3$.

Basically, this scheme is obtained by replacing $\nabla u$ by $P_{s_{1}, \ldots, s_{N}}$ in equation (4) and by choosing the simplex (i.e. $\left.\left(s_{1}, . ., s_{N}\right)\right)$ which contains the dynamics of the optimal control. Moreover, we take advantage of this in order to obtain simultaneously and consistently the approximations of the geodesic distance function and of the optimal dynamics. The implementation of this updating procedure (computation of the solution of (5) and of the optimal dynamics) is based on the "separation" of the simplexes. The method can be resumed by computing the solutions $t_{s}$ of the scheme with a dynamics restricted to each simplex $s$, and then by choosing the smallest solution. The associated simplex is then called optimal simplex. See [25] for a complete description of the computation of the $t_{s}$ and of the associated dynamics.

\subsection{Connectivity Measures Computation}

In this section we detail how to compute the connectivity measure $\mathcal{R}(\tilde{x})$ at the step 1 of our global algorithm. At this stage, we already know the optimal dynamics $f_{\tilde{x}}^{*}$, the optimal simplex $\left(\tilde{x}, x_{1}, x_{2}, x_{3}\right)$ (we denote $x_{i}=\tilde{x}+s_{i}(\tilde{x}) h_{i} e_{i}$ where $s_{i}(\tilde{x})$ is the sign of the $i^{t h}$ component of $f_{\tilde{x}}^{*}$ and $h_{1} \times h_{2} \times h_{3}$ is the size of the voxels) and the values $\mathcal{R}\left(x_{i}\right)$ for $i=1 . .3$.

Let $y$ be the intersection of the optimal trajectory with the front. By assuming that the trajectory is locally affine, we have: $y=\tilde{x}+\tau f_{\tilde{x}}^{*}$ where $\tau$ is the time for the trajectory to reach the front, see figure 1-a). As in [24], we can prove that $\tau=1 / \sum_{i=1 . .3} q_{i}$ where $q_{i}$ is the absolute value of the $i^{\text {th }}$ component of $f_{\tilde{x}}^{*}$ divided by $h_{i}$. By assuming that $\mathcal{R}$ is locally affine, we have [24]

$$
\mathcal{R}(y)=\sum_{i=1}^{3} \tau q_{i} \mathcal{R}\left(x_{i}\right) .
$$

Thus by noting that

$$
\mathcal{R}(\tilde{x})=\mathcal{R}(y)+\int_{0}^{\tau} \mathcal{C}\left(\xi_{\tilde{x}}^{*}(t)\right) d t
$$

we obtain

$$
\mathcal{R}(\tilde{x}) \simeq \sum_{i=1}^{3} \tau q_{i} \mathcal{R}\left(x_{i}\right)+\tau \mathcal{C}(\tilde{x}) .
$$

\section{Remark 1.}

1) The approximation of $\mathcal{S}(\tilde{x})$ is obtained exactly in the same way.

2) This scheme can also be obtained by discretizing the equation $\left\langle\nabla_{E} \mathcal{R}(x), f_{x}^{*}\right\rangle_{E}=\mathcal{C}(x)$ (obtained by evaluating $\left.\lim _{\varepsilon \rightarrow 0} \frac{\mathcal{R}\left(x+\varepsilon f_{x}^{*}\right)-\mathcal{R}(x)}{\varepsilon}\right)$ and by slightly modifying the scheme proposed by [1].

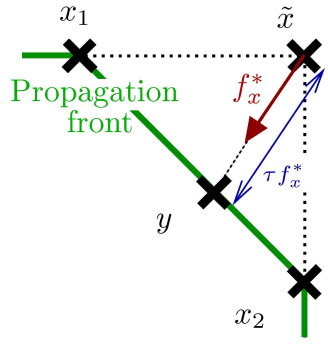

(a) Approximation of the geodesic and localisation of $y$

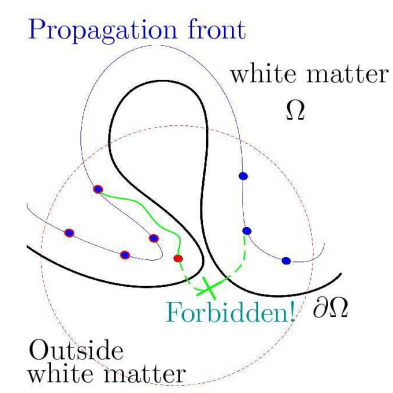

(b) Depiction of the topological problem in a convoluted area of the white matter
Fig. 1.

\section{EXPERIMENTAL RESULTS}

\subsection{Challenging Computational Issues}

The nature of the problem we are trying to solve raises two major computational difficulties which, to our knowledge, are not very well dealt with in the literature.

\subsubsection{Handling the white matter convoluted geometry}

First of all, as illustrated in figure 2 and detailed on figure 1-b), solving the anisotropic eikonal equation within a convoluted domain such as the brain white matter is necessary and complicated. Indeed, the connections we are looking for are defined between cortical areas or between cortical areas and the basal ganglia (a collection of subcortical nuclei deeply included in the white matter). In other words, we are essentially interested in pathways linking together parts of the domain boundary.

In figure 2, the geodesic distance to the blue cross in image (b) (i.e. $x_{0}$ ) was computed, for the DTI data presented in image (a) and within the mask outlined in red in image (b). Its isovalues (in the range $[0,1500]$ ) are depicted by the yellow lines in images (c) and (d). With a level set implementation such as the one proposed by Lenglet et al. [17], the front diffuses through the CSF and directly connects the right hemisphere. This is anatomically incorrect since the fibers starting 


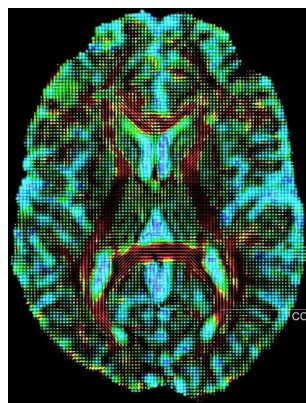

(a) DTI axial slice

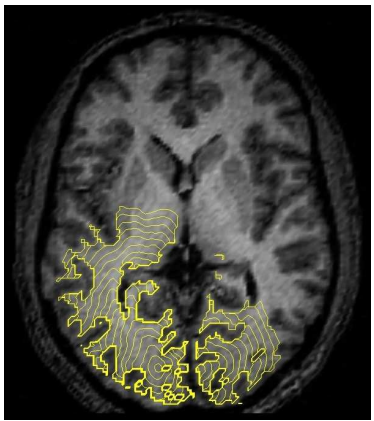

(c) Level set algorithm [17]

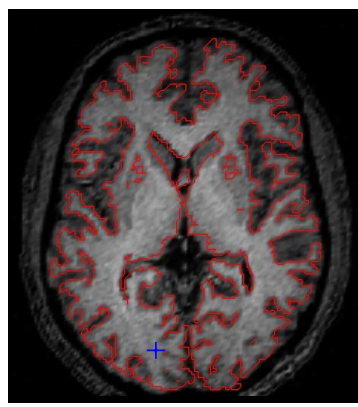

(b) White matter segmentation

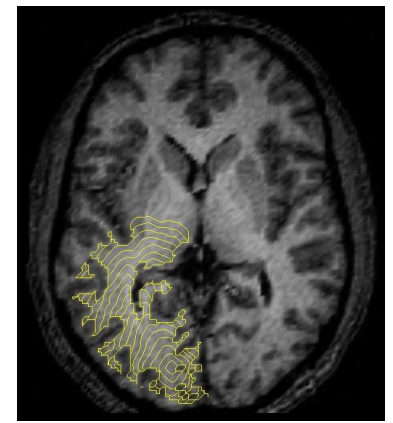

(d) Our new algorithm
Fig. 2. Topological inconsistency in the occipital cortex.

from the blue cross (located in the V1 visual area) go through the corpus callosum (CC) to reach the other hemisphere. Our method correctly estimates the distance since, by definition, it ignores all the locations outside the mask.

This kind of difficulties is also encountered with the Ordered Upwind Method (OUM) recently proposed by Sethian and Vladimirsky [29]. The OUM is a numerical method of type FMM which uses enlarged neighborhoods. The more anisotropic the tensor, the larger the neighborhood. In addition to increasing the computation time, Sethian and Vladimirsky's method explicitly authorizes this type of topological error by allowing the trajectories to step outside the mask and to directly connect any nearby voxel located on the front. The scheme we use here only uses nearest neighbors (six nearest neighbors in 3D). Our method is not sensitive to this problem and always respects the topology of the mask.

\subsubsection{Robust estimation of the optimal dynamics}

The second issue is related to the robustness of the optimal dynamics (i.e. the geodesics tangent vectors) computation. Indeed, all the existing methods need to explicitly compute the derivatives of the distance function. This is well-known to be sensitive to noise, especially on the boundaries where the discretization of the differential needs to be adapted. We present, in figure 3, a comparison of the vector fields obtained by the method proposed in [17] (top row) and by our approach (bottom row) on a 3D synthetic DTI dataset (see image (a);

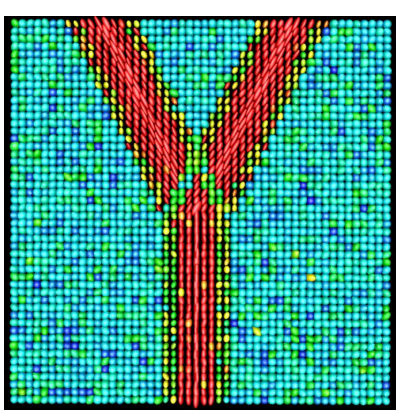

(a) DTI axial slice
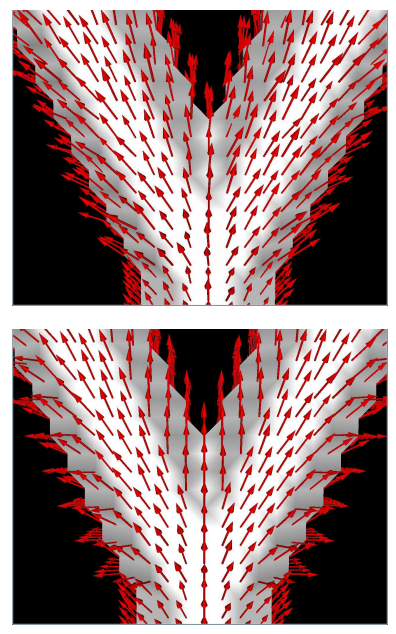

(c) Region A

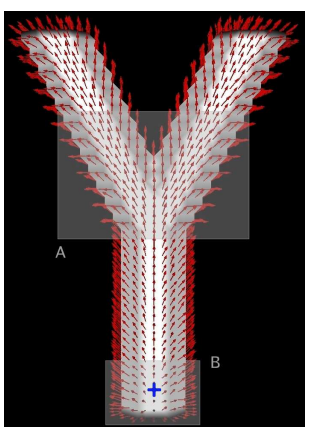

(b) Optimal dynamics
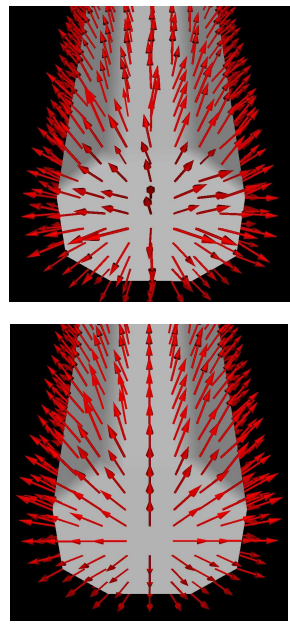

(d) Region B
Fig. 3. Optimal dynamics estimation by differentiation of the distance [(c-d) Top] and by our direct method [(c-d) Bottom].

Anisotropy color code: blue=low/red=high). The origin of the distance function is located at the center of region B (see image (b)).

\subsection{Fast and Robust Anatomical Connectivity Measure}

In the following, we illustrate our method by computing the quantities $\mu$ and $\sigma$, introduced in section 3, as well as the geodesics associated to the highest connectivity measure. This is done on the synthetic tensor field of figure 3 as well as in the splenium (posterior part) of the corpus callosum for the real dataset of figure 2 .

\subsubsection{Data acquisition}

Diffusion weighted images were acquired on a 3 Tesla Bruker scanner at the Centre IRMf de Marseille, France. We used 12 diffusion gradient directions and a $b$-value of $1000 \mathrm{~s} / \mathrm{mm}^{2}$. Acquisitions were repeated 8 times for each direction in order to ensure a good signal-to-noise ratio. Voxel size was $2 \times$ $2 \times 2 \mathrm{~mm}^{3}$ and diffusion tensors were estimated by the robust gradient descent algorithm proposed in [18]. An axial slice of the resulting DT image is presented in figure 2(a). 

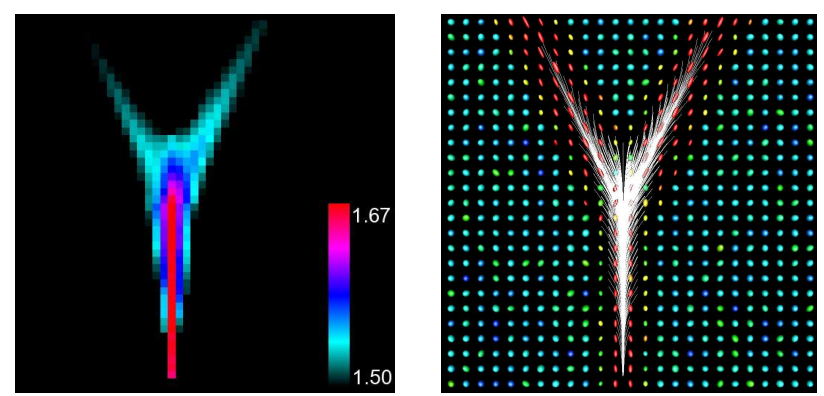

Fig. 4. Synthetic dataset: [Left] Axial slice of the map $\mu$, [Right] Most likely connections.

\subsubsection{Computational efficiency}

PDE methods for brain connectivity mapping such as [20, 23, $17,14,9]$ have the great advantage to yield connectivity information for a point of interest $x_{0}$ to the rest of the brain by exploiting the full information of the diffusion tensor. They are however in general quite time consuming and must be iteratively applied to all the voxels of the functional regions of interest, which can contain hundreds or thousands of points. By comparison with the methods presented in [14] and [17], our algorithm achieves a dramatic improvement in computational speed. For the geodesic distance computation, Jackowski et al. reported a convergence time of about 7 minutes for their iterative sweeping method for a $128 \times 128 \times 40$ DTI dataset on a $1.7 \mathrm{GHz}$ Intel Pentium Xeon with 1.5 $\mathrm{Gb}$ of RAM. We also tested the level set formulation proposed by Lenglet et al. It required about 20 minutes for a $128 \times 128 \times 58$ DTI dataset on a $1.7 \mathrm{GHz}$ Intel Pentium M with $1 \mathrm{~Gb}$ of RAM.

The computation of the geodesics, together with the evaluation of the statistics of $\mathcal{C}(x)$, is itself a time-consuming task since for each curve, we need to explicitly propagate through the tangent vectors field using, for instance, a $4^{\text {th }}$ order Runge-Kutta integration scheme. In [14], no time is given for the computation of the 14, 952 fibers of interest. However, on our data and for 135, 029 voxels inside the white matter mask, it took approximately $\mathbf{3 0}$ minutes on the same computer than the one used for the distance computation. All these computations (distance, vector field and connectivity measures) take about $\mathbf{7}$ seconds with our method.

\subsubsection{Performance of the connectivity measure}

We now demonstrate how the statistics of the quantity $\mathcal{C}(x)$ can be used to evaluate the degree of connectivity of pairs of voxels. First of all, we use the synthetic dataset of figure 3 . The point of interest $x_{0}$ is again located at the center of region B (see image (b)). Figure 4 [Left] presents an axial slice of the thresholded map $\mu$ which is consistent with the DT image since we can see that $\mu$ is higher along the centerline of the $Y$ shape where the tensors are more anisotropic. More-
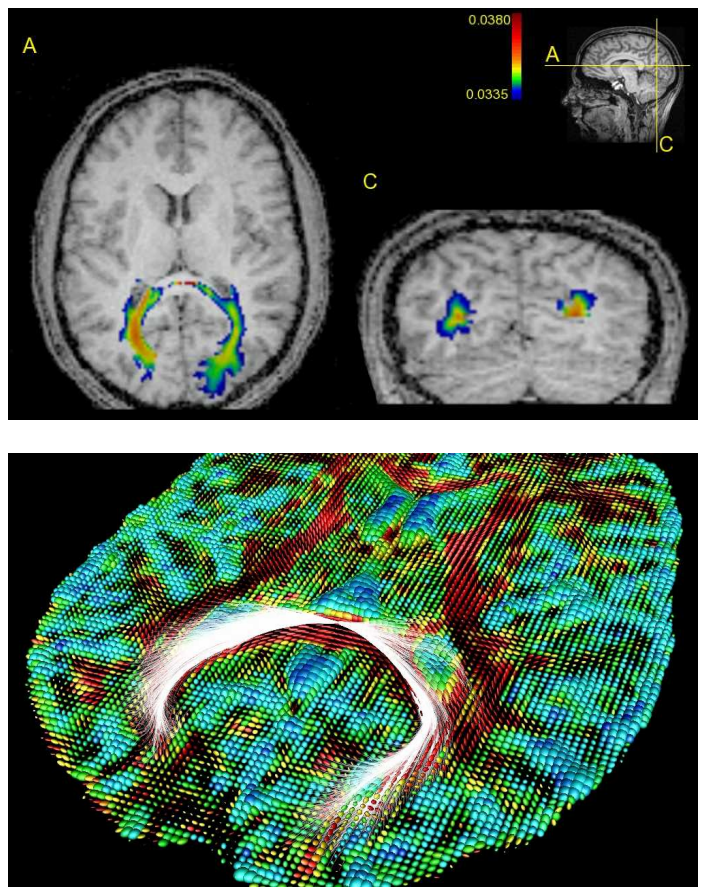

Fig. 5. Real dataset: [Top] Axial and coronal slices of the map $\mu$, [Bottom] Most likely connections (Anisotropy color code: blue=low/red=high).

over, the right branch is clearly more connected to the origin. This is due to the asymmetry imposed by the tensor field in the diverging region (see figure 3 (a)). In figure 4 [Right], we show the geodesics computed from the 873 voxels with values of $\mu$ in the range $[1.5,1.67]$, i.e. the $10 \%$ most likely connected voxels. Finally, we consider the real dataset of figure 5. The origin is located in the middle of the splenium of the corpus callosum. A first threshold is applied on the map $\sigma$ in order to keep only coherent fibers. This yields a binary mask (threshold value: 0.0056 ) which is applied to the map $\mu$. As previously, we then threshold this map to preserve only the top $10 \%$ most likely connected voxels, with values of $\mu$ in the range [0.0335, 0.0380]. This yields 2561 fibers that are consistent with neuro-anatomical knowledge.

\section{CONCLUSION}

We have introduced a general local connectivity measure and experimentally demonstrated its relevance on real data sets. Exploiting both an optimal control and a Riemannian interpretation, we achieved a number of improvements over existing methods. We proposed a fast algorithm that reduces CPU time by 2 or 3 orders of magnitude relatively to existing work. Our algorithm is numerically stable and efficient, since it simultaneously computes the distance function, the optimal dynamics and the statistics of our local connectivity measure from the DT images. Finally we showed that our method overcomes numerical limitations that cause existing 
algorithms to fail in highly convoluted regions. The $\mathrm{C}++\mathrm{im}-$ plementation of our GCM algorithm will be soon freely distributed on the web.

\section{Acknowledgments}

This research was partially supported by grants NIH U54 RR021813, ONR N00014-03-1-0850, AFOSR F49620-03-10095, ACI Obs-Cerv and the Région Provence-Alpes-Côte d'Azur. The authors would like to thank J.L. Anton, M. Roth and N. Wotawa for the human brain DTI dataset used in this paper.

\section{REFERENCES}

[1] D. Adalsteinsson and J. Sethian. The fast construction of extension velocities in level set methods. Journal of Computational Physics, 148(1):2-22, 1999.

[2] A. Alexander, H. K.M., M. Lazar, J. Tsuruda, and D. Parker. Analysis of parial volume effects in diffusion-tensor mri. Magnetic Resonance in Medicine, 45(5):770-780, 2001.

[3] M. Bardi and I. Capuzzo-Dolcetta. Optimal control and viscosity solutions of HJB equations. Birkhauser, 1997.

[4] P. Basser, J. Mattiello, and D. LeBihan. MR diffusion tensor spectroscopy and imaging. BiophysicalJ., 66:259-267, 1994.

[5] P. Basser, S. Pajevic, C. Pierpaoli, J. Duda, and A. Aldroubi. In vivo fiber tractography using dt-mri data. Magn. Res. Med., 44:625-632, 2000.

[6] T. Behrens, M. Woolrich, M. Jenkinson, H. Johansen-Berg, R. Nunes, S. Clare, P. Matthwes, J. Brady, and S. Smith. Characterization and propagation of uncertainty in diffusionweighted $\mathrm{mr}$ images. Magnetic Resonance in Medicine, 50:1077-1088, 2003.

[7] D. L. Bihan, E. Breton, D. Lallemand, P. Grenier, E. Cabanis, and M. Laval-Jeantet. Mr imaging of intravoxel incoherent motions: Application to diffusion and perfusion in neurologic disorders. Radiology, pages 401-407, 1986.

[8] M. Bjornemo, A. Brun, R. Kikinis, and C. Westin. Regularized stochastic white matter tractography using diffusion tensor mri. In MICCAI, pages 435-442, 2002.

[9] S. Campbell, K. Siddiqi, V. Rymar, A. Sadikot, and G. Pike. Flow-based fiber tracking with diffusion tensor q-ball data: Validation and comparison to principal diffusion direction techniques. NeuroImage, 27(4):725-736, Oct. 2005.

[10] L. Cohen. Minimal paths and fast marching methods for image analysis. In Mathematical Models in Computer Vision: The Handbook, chapter 7. Springer, 2005.

[11] M. P. DoCarmo. Riemannian Geometry. Birkhäuser, 1992.

[12] O. Friman and C. Westin. Uncertainty in white matter fiber tractography. In Proceedings of MICCAI'05, 2005.

[13] P. Hagmann, J. Thiran, L. Jonasson, P. Vandergheynst, S. Clarke, P. Maeder, and R. Meuli. Dti mapping of human brain connectivity: Statistical fiber tracking and virtual dissection. NeuroImage, 19:545-554, 2003.

[14] M. Jackowski, C. Kao, M. Qiu, R. Constable, and L. Staib. White matter tractography by anisotropic wavefront evolution and diffusion tensors imaging. Medical Image Analysis, 9:427440, 2005.
[15] R. Kimmel and J. A. Sethian. Computing Geodesic Paths on Manifolds. Proc. of the Nat. Academy of Science, 95, 1998.

[16] M. Lazar, D. Weinstein, J. Tsuruda, K. Hasan, K. Arfanakis, M. Meyerand, B. Badie, H. Rowley, V. Haughton, and A. Alexander. White matter tractography using diffusion tensor deflection. In $H B M, 2003$.

[17] C. Lenglet, R. Deriche, and O. Faugeras. Inferring white matter geometry from diffusion tensor MRI: Application to connectivity mapping. In ECCV'O4, 2004.

[18] C. Lenglet, M. Rousson, R. Deriche, and O. Faugeras. Statistics on the manifold of multivariate normal distributions: Theory and application to Diffusion Tensor MRI. JMIV, 2005.

[19] S. Mori, B. Crain, V. Chacko, and P. V. Zijl. Three-dimensional tracking of axonal projections in the brain by magnetic resonance imaging. An. of Neurology, 45(2):265-269, 1999.

[20] L. O'Donnell, S. Haker, and C. Westin. New approaches to estimation of white matter connectivity in diffusion tensor mri: Elliptic pdes and geodesics in a tensor-warped space. In $M I C$ CAI, 2002. 459-466.

[21] S. Osher and J. Sethian. Fronts propagating with curvaturedependent speed: Algorithms based on Hamilton-Jacobi formulations. J. Computational Physics, 79(1):12-49, 1988.

[22] G. Parker, H. Haroon, and C. Wheeler-Kingshott. A framework for a streamline-based probabilistic index of connectivity (pico) using a structural interpretation of mri diffusion measurements. Journal of Magnetic Resonance Imaging, 18(2):242-254, Aug. 2003.

[23] G. Parker, C. Wheeler-Kingshott, and G. Barker. Estimating distributed anatomical connectivity using fast marching methods and diffusion tensor imaging. Trans. Med. Imaging, 21(5):505-512, 2002.

[24] E. Prados, O. Faugeras, and E. Rouy. Shape from shading and viscosity solutions. Technical Report 4638, INRIA, 2002.

[25] E. Prados, C. Lenglet, J.-P. Pons, N. Wotawa, R. Deriche, O. Faugeras, and S. Soatto. Control theory and fast marching methods for brain connectivity mapping. Technical Report 5845, INRIA, Feb. 2006.

[26] E. Prados and S. Soatto. Fast marching method for generic shape from shading. In VLSM'05, 2005.

[27] E. Rouy and A. Tourin. A Viscosity Solutions Approach to Shape-from-Shading. SIAM J. of Num. Anal., 29(3):867-884, 1992.

[28] J. Sethian. A fast marching sevel set method for monotonically advancing fronts. In Proc. of the Nat. Academy of Sciences, volume 93, pages 1591-1694, 1996.

[29] J. Sethian and A. Vladimirsky. Ordered upwind methods for static hamilton-jacobi equations: Theory and algorithms. SIAM J. on Num. Anal., 41(1):325-363, 2003.

[30] H. M. Soner. Optimal control with state space constraints. SIAM J. Contr. Optim, 24:Part I: 552-562, Part II: 1110-1122, 1986.

[31] Y.-H. Tsai, L.-T. Cheng, S. Osher, and H.-K. Zhao. Fast sweeping algorithms for a class of hamilton-jacobi equations. SIAM J. Numer. Anal., 41(2):673-694, 2003.

[32] J. Tsitsiklis. Efficient algorithms for globally optimal trajectories. IEEE Tr. Aut. Control, 40:1528-1538, 1995.

[33] B. Vemuri, Y. Chen, M. Rao, T. McGraw, T. Mareci, and Z. Wang. Fiber tract mapping from diffusion tensor mri. In Proc. of VLSM'01, July 2001. 